\title{
PARASITISM PERCENTAGES ON APHIS CRACCIVORA KOCH. ON FABA BEAN AND COWPEA PLANTS IN NEWLY RECLAIMED LAND IN EGYPT
}

\author{
Ali, SH. A.M. \\ Plant Prot. Res. Institute, ARC, Dokki, Giza , Egypt \\ (Manuscript received 9 March 2014)
}

\begin{abstract}
The population densities of the cowpea aphid, Aphis craccivora Koch on faba bean and cowpea plants and its parasitoids were studied at El-Khattara district, Sharkia Governorate, Egypt in 2010 - 2011 and 2011-2012 seasons. It recorded three peaks on faba bean and cowpea plants every season. A. craccivora on faba bean was associated with three primary parasitoids being Diaeretiella rapae (M'Intosh), Aphidius colemani Viereck and Ephedrus sp. and only one Hyperparasitoid, Aphidencertus sp.. Primary parasitoid $D$. rapae was the highly dominant species by relative density 54.15 and $51.99 \%$, followed by $A$. colemani 25.65 and $25.43 \%$, Ephedrus sp. (14.05 and $14.07 \%$ ), while the hyperparasitoid Aphidencertus sp., occurred by 6.15 and $8.51 \%$, during 2010 /2011 and 2011 /2012 seasons, respectively. A. craccivora on cowpea was associated with only two primary parasitoids species Lysiphlebus fabarum and Trioxys sp., only one hyperparasitoid species of the family Pteromalidae. Primary parasitoid L., fabarum was the most dominant species by relative density 56.22 and $61.24 \%$, while Trioxys sp. was recorded by 26.32 and $21.48 \%$ and Pteromalidae by 17.46 and $17.28 \%$ during 2010 and 2011 season respectively. Highest rates of parasitism $21.67 \%$ were recorded in the $2^{\text {nd }}$ week of February $2010-2011$ and $22.34 \%$ in the $4^{\text {th }}$ week of February 2011-2012 on faba bean plants, meanwhile it were $20.37 \%$ and $11.37 \%$ in the first week of September 2010 and in the $2^{\text {nd }}$ week of September during the two season cowpea plants. The seasonal means of parasitism rates were ( 9.80 and $8.78 \%$ ), on faba bean plants during two seasons, respectively. While it obtained 8.14 and $4.68 \%$ on cowpea plants during the two season, respectively. Total developmental period of $L$. fabarum was investigated at three temperatures $\left(10,20\right.$ and $\left.30^{\circ} \mathrm{C}\right)$. The duration was longest at $10^{\circ} \mathrm{C}$. Generally, developmental periods of different stages of the parasitoid shorted as the temperature increased from 10 to $30^{\circ} \mathrm{C}$, total developmental period ranged between 8.81 and 21.54 days, respectively.

Key words: Estimation, Parasitism, Aphis craccivora, Faba Bean, Cowpea
\end{abstract}

\section{INTRODUCTION}

Aphids are considered a group of insect pests of which the economic importance increases with the development of agriculture (Stary , 1976). Faba bean is an essential food crop that provides a major source of protein for humans and domestic animals. Cowpea is mainly cultivated for local consumption, either at green 
shell stage for fresh market or at mature stage for dry seeds. Cowpea aphid, Aphis craccivora Koch. is considered one of the most injurious pests infesting faba bean and cowpea plants in Egypt (Selim et al., 1987, El-Defrawi et al. 2000, Ragab et al. 2002 and Saleh 2004 and 2009). It suck the plant sap causing a decrease in plant vitality, and consequently reduce the crop yield. In addition, aphids are considered the most serious vectors of plant viruses which may induce destruction of the infested crop. Biological control is a main factor of integrated control programs including parasitoids, predators and pathogens Sarhan 1976 and Maghraby, 2012 in Egypt, recorded. The parasitoid Diaeretiella rapae (M'Intosh) was, for the first time, recorded in association with the field population of $A$. craccivora, infesting broad bean in Mansoura district. Also, Ragab et al. (2002a), in Egypt, found the three parasitoids D. rapae, Lysiphlebus fabarum (Marshall) and Ephedrus spp. It also parasitized many aphid species, Myzus persicae, Pentalonia nigronervosa, Acyrthosiphon pisum, Sitobion avenae, Shizaphis graminum and Aulacorthum solani (Stary and Stechmann, 1990). Therefore, the present study aims to throw some lights on the parasitoids species associated with aphids infested faba bean and cowpea plants. Also estimation of parasitism rate in the cultivated fields in newly reclaimed sandy areas and life cycle of $L$. fabarum on $A$. craccivora at 10,20 and $30^{\circ} \mathrm{C}$ at $65 \pm 5 \%$ R.H..

\section{MATERIALS AND METHODS}

\section{Survey and estimation of parasitism in the field:}

Field studies were carried out during 2010/2011 and 2011/2012 on faba bean plants and (2011 \& 2012) seasons on cowpea plants, at El-Khattara district, Sharkia Governorate. A. craccivora population was estimated on faba bean plants through weekly random samples of twenty aphids infested leaves of faba bean and cowpea. Samples were transported to the laboratory and all aphid individuals on leaves / sample were counted. Aphids were supplied with fresh host leaves and kept in Petri dishes (50 aphids / Petri dish) until formation of mummies. The emerged parasitoids were mounted and identified by Prof. Dr. A. El-Heneidy, Biological Control Department, Plant Protection Research Institute, Agricultural Research Center, Giza, Egypt. Rates of parasitism and hyperparasitism were estimated according to Farrell and Stufkens (1990) as:

Percentage of parasitism $=\frac{\mathrm{A}+\mathrm{B}}{\mathrm{A}+\mathrm{B}+\mathrm{C}} \times 100$ Where:

$A=$ Number of mummified aphids counted at the date of inspection.

$B=$ Number of mummified host appeared during the laboratory rearing

$\mathrm{C}=$ No of unparasitized aphids. 


\section{Biological studies}

\subsection{Life cycle of Lysiphlebus fabarum on nymphs $A$. craccivora}

A laboratory culture of $A$. craccivora was maintained under laboratory conditions. Nymphs of $A$. craccivora were reared on caged young seedlings of $V$. faba grown after sowing in wet sawdust. A laboratory culture of $L$. fabarum started with mummies obtained from the field. Mummified aphids were placed singly in small glass tubes until emergence of adult parasitoid which were fed on sugar solution (30\%). To determine the durations of different immature stages of $L$. fabarum on nymphs $A$. craccivora at three temperatures $\left(10,20\right.$ and $\left.30^{\circ} \mathrm{C}\right)$, nymphs were confined with the parasitoid in a chimney glass for four hours. Thirty nymphs of parasitized host aphids were daily dissected to determine the development of different immature stages of the parasitoid.

\subsection{Sex ratio}

Sex ratio of $L$. fabarum recovered from $A$. craccivora was determined and obtained data were statistically analyzed using, Costat (1990) computer program.

\section{RESULTS AND DISCUSSION}

\section{Survey and estimation of parasitism in the faba bean and cowpea fields}

\subsection{Survey and estimation of parasitism in the faba bean field}

The weekly counts of aphids on faba bean plants during 2010/2011 and 2011/2012 seasons are given in (Tables 1 and 2). During the first season, the infestation started to appear on the $3^{\text {rd }}$ week of November ( 24.9 and $65.3 \%$ R.H.), while appeared during the $3^{\text {rd }}$ week of November $\left(24.7^{\circ} \mathrm{C}\right.$ and $61.8 \%$ R.H.) in the second season.

The following is a list of hymenopterous primary and hyper parasitoid species that emerged from cowpea aphid $A$. craccivora during the period of study:

\section{On faba bean}

Primary parasitoids: Diaeretiella rapae (M'Intosh) Aphidius colemani Viereck and Ephedrius sp.

Hyperparasitoids: Aphidencyrtus sp.

On cowpea

Primary parasitoids: Lysiphelous fabarum and Trioxys sp. Hyperparasitoid: Belong to family Pteromalidae. 
Table 1. Survey and relative densities of Aphis craccivora Koch. parasitoids on faba bean and cowpea plants cultivated in the newly reclaimed sandy area of El-Khattara district, Sharkia Governorate, Egypt during two successive seasons.

\begin{tabular}{|c|c|c|c|c|c|}
\hline \multirow{3}{*}{ Species } & \multirow{3}{*}{ Family } & \multicolumn{4}{|c|}{ Faba bean } \\
\hline & & \multicolumn{2}{|c|}{$2010 / 2011$} & \multicolumn{2}{|c|}{$2011 / 2012$} \\
\hline & & Number & $\%$ & Number & $\%$ \\
\hline \multicolumn{6}{|l|}{ Primary parasitoids: } \\
\hline Diaeretiella rapae (M'Intosh) & Aphidiidae & 555 & 54.15 & 458 & 51.99 \\
\hline Aphidius colemani Viereck & & 263 & 25.65 & 224 & 25.43 \\
\hline Ephedrus persica & & 144 & 14.05 & 124 & 14.07 \\
\hline \multicolumn{6}{|l|}{ Hyperparasitoids: } \\
\hline Aphidencertus sp. & Encyrtidae & 63 & 6.15 & 75 & 8.51 \\
\hline \multirow[t]{4}{*}{ Total } & & 1025 & 100 & 881 & 100 \\
\hline & & \multicolumn{4}{|c|}{ Cowpea } \\
\hline & & \multicolumn{2}{|c|}{2010} & \multicolumn{2}{|c|}{2011} \\
\hline & & Number & $\%$ & Number & $\%$ \\
\hline \multicolumn{6}{|l|}{ Primary parasitoids: } \\
\hline Lysiphlebus fabarum (Mars.) & Aphidiidae & 425 & 56.22 & 248 & 61.24 \\
\hline Trioxys sp. & & 199 & 26.32 & 87 & 21.48 \\
\hline \multicolumn{6}{|l|}{ Hyperparasitoids: } \\
\hline Unideritified species & Pteromalidae & 132 & 17.46 & 70 & 17.28 \\
\hline Total & & 756 & 100 & 405 & 100 \\
\hline
\end{tabular}


Table 2. Parasitism percentage of Aphis craccivora Koch. infested faba bean plants, cultivated in El-Khattara district, Sharkia Governorate, Egypt during 2010/2011 season.

\begin{tabular}{|c|c|c|c|c|c|c|c|c|c|c|c|c|c|c|c|c|}
\hline \multirow{4}{*}{$\begin{array}{l}\text { Sampling date } \\
\text { (weeks) }\end{array}$} & \multirow{4}{*}{ 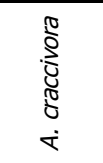 } & \multirow{2}{*}{\multicolumn{3}{|c|}{$\begin{array}{l}\text { No. of parasitized } \\
\text { aphid (mummies) }\end{array}$}} & \multirow{4}{*}{ 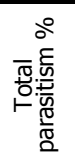 } & \multicolumn{8}{|c|}{ Emerged parasitoids } & \multirow{4}{*}{ Total } & \multirow{4}{*}{$\begin{array}{l}\text { Mean } \\
\text { oC }\end{array}$} & \multirow{4}{*}{ Mean R.H. } \\
\hline & & & & & & \multirow{2}{*}{\multicolumn{2}{|c|}{ D.rapae }} & \multirow{2}{*}{\multicolumn{2}{|c|}{$\begin{array}{c}\text { Primary parasitoids } \\
\text { A. colemani }\end{array}$}} & & & \multirow{2}{*}{\multicolumn{2}{|c|}{ Aphidencyrtus sp. }} & & & \\
\hline & & \multirow{2}{*}{ A } & \multirow{2}{*}{ B } & \multirow{2}{*}{ Total } & & & & & & \multicolumn{2}{|c|}{ Ephedrius sp. } & & & & & \\
\hline & & & & & & No. & $\%$ & No. & $\%$ & No. & $\%$ & No. & $\%$ & & & \\
\hline Nov. $3^{\text {rd }}$ & 230 & 0 & 0 & 0 & 0 & 0 & 0 & 0 & 0 & 0 & 0 & 0 & 0 & 0 & 24.9 & 65.3 \\
\hline $4^{\text {th }}$ & 345 & 0 & 5 & 5 & 1.45 & 3 & 100 & 0 & 0 & 0 & 0 & 0 & 0 & 3 & 23.7 & 65.3 \\
\hline Dec. $1^{\text {st }}$ & 450 & 4 & 8 & 12 & 2.67 & 5 & 50 & 3 & 30 & 2 & 20 & 0 & 0 & 10 & 22.1 & 70.8 \\
\hline $2^{\text {nd }}$ & 775 & 12 & 19 & 31 & 4.0 & 14 & 53.85 & 8 & 30.77 & 4 & 15.38 & 0 & 0 & 26 & 21.7 & 61.6 \\
\hline $3^{\text {rd }}$ & 840 & 14 & 38 & 52 & 6.19 & 29 & 64.45 & 10 & 22.22 & 6 & 13.33 & 0 & 0 & 45 & 22.1 & 64.8 \\
\hline $5^{\text {th }}$ & 2435 & 105 & 125 & 230 & 9.45 & 102 & 52.31 & 68 & 34.87 & 25 & 12.82 & 0 & 0 & 195 & 21.4 & 73.3 \\
\hline \begin{tabular}{|l} 
Jan. \\
$1^{\text {st }}$
\end{tabular} & 902 & 35 & 90 & 125 & 13.86 & 68 & 63.55 & 24 & 22.43 & 15 & 14.02 & 0 & 0 & 107 & 22.2 & 68.6 \\
\hline $2^{\text {nd }}$ & 654 & 34 & 85 & 119 & 18.20 & 64 & 64.65 & 22 & 22.22 & 13 & 13.13 & 0 & 0 & 99 & 21.2 & 64.6 \\
\hline $3^{\text {rd }}$ & 580 & 30 & 52 & 82 & 14.14 & 40 & 60.61 & 15 & 22.73 & 8 & 12.12 & 3 & 4.54 & 66 & 20.6 & 65.6 \\
\hline $4^{\text {th }}$ & 320 & 8 & 23 & 31 & 9.69 & 12 & 44.45 & 7 & 25.93 & 4 & 14.81 & 4 & 14.81 & 27 & 20.1 & 63.3 \\
\hline Feb. $1^{\text {st }}$ & 240 & 5 & 19 & 24 & 10.0 & 6 & 28.57 & 4 & 19.05 & 3 & 14.28 & 8 & 38.10 & 21 & 19.5 & 63.5 \\
\hline $2^{\text {nd }}$ & 420 & 38 & 53 & 91 & 21.67 & 42 & 52.50 & 22 & 27.50 & 12 & 15.0 & 4 & 5.0 & 80 & 19.4 & 58.3 \\
\hline $3^{\text {rd }}$ & 214 & 16 & 24 & 40 & 18.69 & 14 & 43.75 & 6 & 18.75 & 4 & 12.50 & 8 & 25.0 & 32 & 25.6 & 60.8 \\
\hline $2^{\text {nd }}$ & 315 & 22 & 28 & 50 & 15.87 & 12 & 30.77 & 14 & 35.90 & 8 & 20.51 & 5 & 12.82 & 39 & 25.9 & 61.7 \\
\hline $3^{\text {rd }}$ & 205 & 10 & 16 & 26 & 12.68 & 7 & 36.84 & 5 & 26.32 & 4 & 21.05 & 3 & 15.79 & 19 & 26.7 & 65.6 \\
\hline $4^{\text {th }}$ & 182 & 3 & 10 & 13 & 7.14 & 5 & 50 & 3 & 30 & 0 & 0 & 2 & 20.0 & 10 & 21.8 & 63.7 \\
\hline $5^{\text {th }}$ & 140 & 0 & 8 & 8 & 5.71 & 3 & 60 & 2 & 40 & 0 & 0 & 0 & 0 & 5 & 23.9 & 59.7 \\
\hline Apr. $\quad 1^{\text {st }}$ & 63 & 0 & 0 & 0 & 0 & 0 & 0 & 0 & 0 & 0 & 0 & 0 & 0 & 0 & 27.3 & 57.0 \\
\hline Total & 12216 & & & & & 555 & 54.15 & 263 & 25.66 & 144 & 14.05 & 63 & 6.14 & 1025 & & \\
\hline Mean & $\begin{array}{l}581.71+ \\
127.41\end{array}$ & & & & $\begin{array}{l}9.80+ \\
1.36 \\
\end{array}$ & $\begin{array}{r}26.43 \pm \\
6.6 \pm\end{array}$ & $\begin{array}{r}47.45 \pm \\
4.9 \pm \\
\end{array}$ & $\begin{array}{r}12.52+ \\
3.28\end{array}$ & $\begin{array}{r}22.59 \pm \\
2.41^{-} \\
\end{array}$ & $\begin{array}{r}6.86+ \\
1.47 \\
\end{array}$ & $\begin{array}{c}11.71+ \\
1.58 \\
\end{array}$ & $\begin{array}{l}3.0+ \\
0.94 \\
\end{array}$ & $\begin{array}{r}8.73+ \\
2.52 \\
\end{array}$ & $\begin{array}{r}48.81+ \\
10.96 \\
\end{array}$ & & \\
\hline
\end{tabular}


However, Selim et al. (1987), reported that $A$. craccivora was parasitized by Aphidius colemani and L. fabarum in faba bean fields, in 1996 Abdel -Samad recorded L. fabarum as a parasitoid on A. craccivora in Egypt. Moreover, AbdelSamad (1996), Ragab (1996) and Saleh (2009) recorded Trioxys angelicae (Hal.) as parasitoid of $A$. craccivora.

The present results agree with those of Ragab et al. (2002b), Saleh (2009) and Maghraby (2012), who found that D. rapae, L. fabarum and Ephedrus sp. as parasitoids on A. craccivora in Egypt. Abdel-Samad and Ahmed (2006), recorded $L$. fabarum, A. matricariae and Trioxys sp. were found attacking $A$. craccivora on faba bean cultivars.

\section{On faba bean plants}

The primary parasitoid $D$. rapae was the most dominant species with mean relative densities ( 54.15 and $51.99 \%$ ) during the two seasons respectively. Meanwhile, the mean relative densities of $A$. colemani were 25.65 and $25.43 \%$ respectively, but Ephedrus sp., recorded 14.05 and $14.07 \%$, respectively. The hyperparasitoid Aphidencertus sp. recorded 6.15 and $8.51 \%$ respectively, (Table 1 ).

The percentage of parasitism ranged from 1.45 to 21.67 , at the ranges of 23.7 \& $19.4^{\circ} \mathrm{C}$ and $65.3 \& 58.3 \%$ R.H., respectively, in the first season $2010 / 2011$, Table (2). In the second season, percentage of parasitism ranged from 2.50 to $22.34 \%$ on the $4^{\text {th }}$ week of November at $21.8^{\circ} \mathrm{C}$ and $61.2 \%$ R.H. (Table, 3). Highest percentage of parasitism reached $22.34 \%$ on the $4^{\text {th }}$ week of February at $19.4^{\circ} \mathrm{C}$ and $60.7 \%$ R.H. Total means of parasitism rates by $D$. rapae, A. colemani, Ephedrius sp. and Aphidencyrtus sp. were 9.80 and $8.78 \%$ during $2010 / 2011$ and 2011/2012 seasons, respectively, (Tables 2 and 3). 
Table 3. Parasitism percentage of Aphis craccivora Koch. infested faba bean plants cultivated in El-Khattara district, Sharkia Governorate, Egypt during 2011/2012 season.

\begin{tabular}{|c|c|c|c|c|c|c|c|c|c|c|c|c|c|c|c|c|}
\hline \multirow{4}{*}{\begin{tabular}{|c|} 
Sampling date \\
(weeks)
\end{tabular}} & \multirow{4}{*}{$+\frac{\substack{0 \\
0}}{0}$} & \multirow{2}{*}{\multicolumn{3}{|c|}{$\begin{array}{c}\text { No. of parasitized aphid } \\
\text { (mummies) }\end{array}$}} & \multirow{4}{*}{ 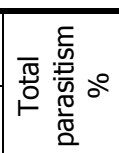 } & \multicolumn{8}{|c|}{ Emerged parasitoids } & \multirow{4}{*}{ Total } & \multirow{4}{*}{$\begin{array}{l}\text { Mean } \\
{ }^{\circ} \mathrm{C}\end{array}$} & \multirow{4}{*}{ Mean R.H } \\
\hline & & & & & & \multirow{2}{*}{\multicolumn{2}{|c|}{ D.rapae }} & \multirow{2}{*}{\multicolumn{2}{|c|}{$\begin{array}{c}\text { Primary parasitoids } \\
\text { A. colemani }\end{array}$}} & & & \multirow{2}{*}{\multicolumn{2}{|c|}{$\begin{array}{c}\text { Hyperparasitoid } \\
\text { Aphidencyrtus sp. }\end{array}$}} & & & \\
\hline & & \multirow[t]{2}{*}{\begin{tabular}{|l|}
$A$ \\
\end{tabular}} & \multirow[t]{2}{*}{$B$} & \multirow[t]{2}{*}{ Total } & & & & & & \multicolumn{2}{|c|}{ Ephedrius sp. } & & & & & \\
\hline & & & & & & No. & $\%$ & No. & $\%$ & No. & $\%$ & No. & $\%$ & & & \\
\hline Nov. $3^{\text {rd }}$ & 160 & 0 & 0 & 0 & 0 & 0 & 0 & 0 & 0 & 0 & 0 & 0 & 0 & 0 & 24.7 & 61.8 \\
\hline $4^{\text {th }}$ & 320 & 0 & 8 & 8 & 2.50 & 6 & 100 & 0 & 0 & 0 & 0 & 0 & 0 & 6 & 21.8 & 61.2 \\
\hline Dec. $1^{\text {st }}$ & 458 & 0 & 12 & 12 & 2.62 & 9 & 100 & 0 & 0 & 0 & 0 & 0 & 0 & 9 & 22.3 & 66.4 \\
\hline $2^{\text {nd }}$ & 615 & 3 & 14 & 17 & 2.76 & 11 & 73.34 & 2 & 13.33 & 2 & 13.33 & 0 & 0 & 15 & 19.8 & 61.6 \\
\hline $3^{\text {rd }}$ & 714 & 12 & 23 & 35 & 4.90 & 20 & 64.51 & 8 & 25.81 & 3 & 9.68 & 0 & 0 & 31 & 16.5 & 57.8 \\
\hline $4^{\text {th }}$ & 820 & 21 & 25 & 46 & 5.61 & 24 & 60 & 12 & 30 & 4 & 10 & 0 & 0 & 40 & 16.9 & 59.6 \\
\hline $5^{\text {th }}$ & 942 & 30 & 48 & 78 & 8.28 & 33 & 49.25 & 22 & 32.84 & 12 & 17.91 & 0 & 0 & 67 & 17.6 & 51.0 \\
\hline Jan. $\quad 1^{\text {st }}$ & 1530 & 45 & 84 & 129 & 8.43 & 55 & 52.38 & 35 & 33.33 & 15 & 14.29 & 0 & 0 & 105 & 16.6 & 60.8 \\
\hline $2^{\text {nd }}$ & 840 & 20 & 30 & 50 & 5.95 & 21 & 48.84 & 12 & 27.91 & 10 & 23.25 & 0 & 0 & 43 & 16.8 & 61.4 \\
\hline $3^{\text {rd }}$ & 720 & 18 & 31 & 49 & 6.81 & 20 & 47.62 & 14 & 33.33 & 8 & 19.05 & 0 & 0 & 42 & 15.2 & 60.0 \\
\hline $4^{\text {th }}$ & 640 & 16 & 30 & 46 & 7.19 & 18 & 46.15 & 14 & 35.90 & 7 & 17.95 & 0 & 0 & 39 & 17.3 & 60.0 \\
\hline Feb. $1^{\text {st }}$ & 635 & 20 & 34 & 54 & 8.50 & 23 & 52.27 & 13 & 29.55 & 6 & 13.64 & 2 & 4.54 & 44 & 18.1 & 59.6 \\
\hline $2^{\text {nd }}$ & 612 & 25 & 39 & 64 & 10.46 & 27 & 49.09 & 10 & 18.18 & 12 & 21.82 & 6 & 10.91 & 55 & 16.1 & 60.1 \\
\hline $3^{\text {rd }}$ & 414 & 28 & 50 & 78 & 18.84 & 28 & 46.67 & 12 & 20 & 13 & 21.67 & 7 & 11.66 & 60 & 17.3 & 60.2 \\
\hline $4^{\text {th }}$ & 940 & 90 & 120 & 210 & 22.34 & 93 & 51.67 & 47 & 26.11 & 22 & 12.22 & 18 & 10 & 180 & 19.4 & 60.7 \\
\hline Mar. $1^{\text {st }}$ & 310 & 25 & 40 & 65 & 20.97 & 23 & 41.82 & 12 & 21.82 & 5 & 9.09 & 15 & 27.27 & 55 & 19.9 & 60.8 \\
\hline $2^{\text {nd }}$ & 220 & 10 & 19 & 29 & 13.18 & 8 & 40 & 4 & 20 & 3 & 15 & 5 & 25 & 20 & 17.6 & 59.1 \\
\hline $3^{\text {rd }}$ & 375 & 20 & 22 & 42 & 11.20 & 14 & 40 & 3 & 8.57 & 2 & 5.71 & 16 & 45.72 & 35 & 18.0 & 58.4 \\
\hline $4^{\text {th }}$ & 210 & 14 & 16 & 30 & 14.29 & 17 & 68 & 2 & 8 & 0 & 0 & 6 & 24 & 25 & 21.1 & 59.0 \\
\hline $5^{\text {th }}$ & 125 & 0 & 12 & 12 & 9.60 & 8 & 80 & 2 & 20 & 0 & 0 & 0 & 0 & 10 & 20.7 & 57.6 \\
\hline Apr. $1^{\text {st }}$ & 35 & 0 & 0 & 0 & 0 & 0 & 0 & 0 & 0 & 0 & 0 & 0 & 0 & 0 & 21.7 & 59.1 \\
\hline Total & 11635 & 332 & 623 & 955 & & 458 & 51.99 & 224 & 25.43 & 124 & 14.07 & 75 & 8.51 & 881 & & \\
\hline Mean & $\begin{array}{c}554.05 \pm \\
77.04\end{array}$ & & & & $\begin{array}{c}8.78 \pm \\
1.38\end{array}$ & $\begin{array}{c}21.81 \pm \\
4.45\end{array}$ & $\begin{array}{c}52.93 \pm \\
5.39\end{array}$ & $\begin{array}{c}10.67 \pm \\
2.61\end{array}$ & $\begin{array}{c}19.27 \pm \\
2.68\end{array}$ & $\begin{array}{c}5.90 \pm \\
1.34\end{array}$ & $\begin{array}{c}10.70 \pm \\
1.79\end{array}$ & $\begin{array}{c}3.57 \pm \\
1.28\end{array}$ & $\begin{array}{l}7.58 \pm \\
2.76\end{array}$ & $\begin{array}{l}41.95 \pm \\
8.85\end{array}$ & & \\
\hline
\end{tabular}


One hyperparasitoid Aphidencertus sp. was recorded in few numbers from the $3^{\text {rd }}$ week of January, to $4^{\text {th }}$ week of March in the first season, Table (2), and from the first week of February to the $4^{\text {th }}$ week of March, in the second season, Table (3). Total means of hyperparasitism rates were 3.0 and $3.57 \%$ during the two seasons respectively (Tables 2 and 3). However, Abou-Attia et al. (2001) and Saleh et al. (2009) recorded Aphidencyrtus sp. (Encyrtidae), found on faba bean plants.

\subsection{Survey and estimation of parasitism in the cowpea field}

The weekly counts of aphids on cowpea plants during 2010 and 2011 seasons are given in Tables 4 and 5. During the first season the infestation started on the $3^{\text {rd }}$ and $4^{\text {th }}$ week of June at means $30.5^{\circ} \mathrm{C} \& 63.6 \%$ R.H. and $27.8^{\circ} \mathrm{C} \& 59.0 \%$ R.H., respectively.

The following is a list of hymenopterous primary and hyperparasitoid species that emerged from $A$. craccivora during the period of study:

Primary parasitoids: Lysiphlebus fabarum (Mars.) and Trioxys sp.

Hyperparasitoids: One unidentified species of Pteromalidae

The present results agree with those of Stary and Erdelen (1987), in Yemen, who mentioned that $A$. colemani was the dominant parasite reared from $A$. craccivora on Vigna sp., Cruz et al. (1992), in Portugal, mentioned that Diaeretiella sp. and Trioxys sp. were a primary parasitoid of $A$. craccivora in cowpea fields. Youssif (2009), in Egypt , found that the most dominant primary parasitoids were D. rapae, A. colemani, Aphidius sp. and Trioxys sp. and unidentified species of hyperparasitoids belonged to the two families, Pteromalidae and Cynipidae on A. craccivora infested cowpea plants.

\section{On cowpea plants:}

The primary parasitoid Lysiphlebus fabarum was the most dominant species with mean relative densities 56.22 and $61.24 \%$ during 2010 and 2011 seasons respectively. While Trioxys sp. recorded 26.32 and $21.48 \%$, respectively, the Pteromalidae was represented by 17.46 and $17.28 \%$, respectively, (Table 1 ).

Table (4), showed that the percentage of parasitism ranged from 2.35 to $20.37 \%$, on the first week of July and first week of September at means $34.0 \& 29.7^{\circ} \mathrm{C}$ and $67.4 \& 66.0 \%$ R.H., respectively, during the first season. In the second season, (Table 5), percentage of parasitism ranged between 1.09 and $11.37 \%$, on the $4^{\text {th }}$ week of July and $2^{\text {nd }}$ week of September at means $31.4 \& 28.3^{\circ} \mathrm{C}$ and $62.6 \& 61.8 \%$ R.H., respectively. Total means of parasitism rates by L. fabarum, Trioxys $\mathrm{sp}$. and Pteromalidae, recorded 8.14 and $4.68 \%$ during 2010 and 2011 seasons respectively. One species of hyperparasitoid from the family Pteromalidae was recorded in few numbers from $4^{\text {th }}$ week of July to $4^{\text {th }}$ week of September recording three peaks of population density by 10,15 and 30 individuals/ sample on the $5^{\text {th }}$ week of July, $4^{\text {th }}$ week of August and $3^{\text {rd }}$ week of September at means $35.3,31.4$ and $28.3^{\circ} \mathrm{C}$ and 70.1 , 67.0 and $62.6 \%$ R.H. respectively, during the first season (Table , 4). 
Table 4. Parasitism percentage of Aphis craccivora Koch. infested cowpea plants, cultivated in El-Khattara district, Sharkia Governorate, Egypt during 2010

\begin{tabular}{|c|c|c|c|c|c|c|c|c|c|c|c|c|c|c|}
\hline \multirow{4}{*}{$\begin{array}{l}\text { Sampling } \\
\text { date } \\
\text { (weeks) }\end{array}$} & \multirow{4}{*}{ 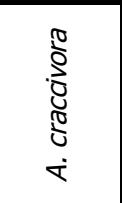 } & \multirow{2}{*}{\multicolumn{3}{|c|}{$\begin{array}{l}\text { No. of parasitized aphid } \\
\text { (mummies) }\end{array}$}} & \multirow{4}{*}{ 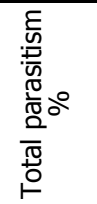 } & \multicolumn{6}{|c|}{ Emerged parasitoids } & \multirow{4}{*}{ Total } & \multirow{4}{*}{$\begin{array}{l}\text { Mean } \\
{ }^{\circ} \mathrm{C}\end{array}$} & \multirow{4}{*}{ Mean R.H } \\
\hline & & & & & & & Primary & sitoids & & \multirow{2}{*}{\multicolumn{2}{|c|}{ Hyperparasitoid Ptyermalidae }} & & & \\
\hline & & \multirow{2}{*}{ A } & \multirow{2}{*}{ B } & \multirow{2}{*}{ Total } & & \multicolumn{2}{|c|}{ Lysiphlebus fabarum } & \multicolumn{2}{|c|}{ Trioxys sp. } & & & & & \\
\hline & & & & & & No. & $\%$ & No. & $\%$ & No. & $\%$ & & & \\
\hline Jun., $3^{\text {rd }}$ & 20 & 0 & 0 & 0 & 0 & 0 & 0 & 0 & 0 & 0 & 0 & 0 & 30.5 & 63.6 \\
\hline $4^{\text {th }}$ & 85 & 0 & 0 & 0 & 0 & 0 & 0 & 0 & 0 & 0 & 0 & 0 & 32.7 & 64.3 \\
\hline Jul., $\quad 1^{\text {st }}$ & 170 & 0 & 4 & 4 & 2.35 & 2 & 100 & 0 & 0 & 0 & 0 & 2 & 34.0 & 67.4 \\
\hline $2^{\text {nd }}$ & 230 & 2 & 5 & 7 & 3.04 & 3 & 60 & 2 & 40 & 0 & 0 & 5 & 32.9 & 64.1 \\
\hline $3^{\text {rd }}$ & 518 & 5 & 18 & 23 & 4.44 & 12 & 66.67 & 6 & 33.33 & 0 & 0 & 18 & 34.2 & 70.0 \\
\hline $4^{\text {th }}$ & 685 & 14 & 25 & 39 & 5.69 & 18 & 62.07 & 9 & 31.03 & 2 & 6.90 & 29 & 34.8 & 70.4 \\
\hline $5^{\text {th }}$ & 845 & 32 & 37 & 69 & 8.17 & 24 & 51.06 & 13 & 27.66 & 10 & 21.28 & 47 & 35.3 & 70.1 \\
\hline Aug., $1^{\text {st }}$ & 514 & 28 & 31 & 59 & 11.48 & 20 & 54.05 & 10 & 27.03 & 7 & 18.92 & 37 & $35 . .4$ & 73.3 \\
\hline $2^{\text {nd }}$ & 416 & 25 & 30 & 55 & 13.22 & 23 & 65.71 & 7 & 20 & 5 & 14.29 & 35 & 36.3 & 73.4 \\
\hline $3^{\text {rd }}$ & 675 & 62 & 36 & 99 & 14.67 & 48 & 51.06 & 14 & 27.66 & 10 & 21.28 & 72 & 34.1 & 72.0 \\
\hline $4^{\text {th }}$ & 1724 & 109 & 150 & 259 & 15.02 & 122 & 67.03 & 45 & 24.73 & 15 & 8.24 & 182 & 31.4 & 67.0 \\
\hline Sep., $1^{\text {st }}$ & 820 & 72 & 95 & 167 & 20.37 & 82 & 63.07 & 30 & 23.08 & 18 & 13.85 & 130 & 29.7 & 66.0 \\
\hline $2^{\text {nd }}$ & 519 & 41 & 52 & 93 & 17.92 & 26 & 36.11 & 25 & 34.72 & 21 & 29.17 & 72 & 28.2 & 64.3 \\
\hline $3^{\text {rd }}$ & 1345 & 83 & 64 & 147 & 10.93 & 32 & 34.78 & 30 & 32.61 & 30 & 32.61 & 92 & 28.3 & 62.6 \\
\hline $4^{\text {th }}$ & 710 & 28 & 15 & 43 & 6.06 & 10 & 31.25 & 8 & 25 & 14 & 43.75 & 32 & 29.7 & 67.8 \\
\hline Oct., $1^{\text {st }}$ & 118 & 4 & 2 & 6 & 5.08 & 3 & 100 & 0 & 0 & 0 & 0 & 3 & 29.1 & 69.0 \\
\hline $2^{\text {nd }}$ & 45 & 0 & 0 & 0 & 0 & 0 & 0 & 0 & 0 & 0 & 0 & 0 & 27.0 & 65.0 \\
\hline Total & 9439 & & & & 138.44 & 425 & 56.22 & 199 & 26.32 & 132 & 17.46 & 756 & & \\
\hline Mean \pm SE & $\begin{array}{c}555.23 \pm \\
112.39 \\
\end{array}$ & & & & $\begin{array}{r}8.14 \pm \\
1.57 \\
\end{array}$ & $\begin{array}{l}25.0 \pm \\
7.92 \\
\end{array}$ & $\begin{array}{r}49.58 \pm \\
7.31 \\
\end{array}$ & $\begin{array}{r}11.71 \pm \\
3.22 \\
\end{array}$ & $\begin{array}{c}20.40 \pm \\
3.48 \\
\end{array}$ & $\begin{array}{r}7.76 \pm \\
2.22 \\
\end{array}$ & $\begin{array}{r}12.37 \pm \\
3.32 \\
\end{array}$ & $\begin{array}{r}44.47 \pm \\
12.47 \\
\end{array}$ & & \\
\hline
\end{tabular}


Table 5. Parasitism percentage of Aphis craccivora Koch. infested cowpea plants, cultivated in El-Khattara district, Sharkia Governorate, Egypt during 2011season.

\begin{tabular}{|c|c|c|c|c|c|c|c|c|c|c|c|c|c|c|}
\hline \multirow{4}{*}{$\begin{array}{c}\text { Sampling } \\
\text { date } \\
\text { (weeks) }\end{array}$} & \multirow{4}{*}{ 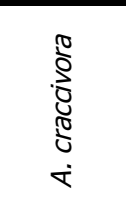 } & \multirow{2}{*}{\multicolumn{3}{|c|}{$\begin{array}{l}\text { No. of parasitized aphid } \\
\text { (mummies) }\end{array}$}} & \multirow{4}{*}{ 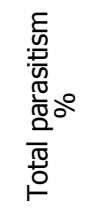 } & \multicolumn{6}{|c|}{ Emerged parasitoids } & \multirow{4}{*}{ Total } & \multirow{4}{*}{$\begin{array}{l}\text { Mean } \\
\text { oC }\end{array}$} & \multirow{4}{*}{ Mean R.H } \\
\hline & & & & & & \multirow{2}{*}{\multicolumn{2}{|c|}{ Lysiphlebus fabarum }} & itoids & & \multirow{2}{*}{\multicolumn{2}{|c|}{ Hyperparasitoid Pteromalidae }} & & & \\
\hline & & \multirow{2}{*}{ A } & \multirow{2}{*}{ B } & \multirow{2}{*}{ Total } & & & & \multicolumn{2}{|c|}{ Trioxys sp. } & & & & & \\
\hline & & & & & & No. & $\%$ & No. & $\%$ & No. & $\%$ & & & \\
\hline Jun., $3^{\text {rd }}$ & 0 & 0 & 0 & 0 & 0 & 0 & 0 & 0 & 0 & 0 & 0 & 0 & 26.5 & 58.4 \\
\hline $4^{\text {th }}$ & 25 & 0 & 0 & 0 & 0 & 0 & 0 & 0 & 0 & 0 & 0 & 0 & 27.8 & 59.0 \\
\hline Jul., $\quad 1^{\text {st }}$ & 72 & 0 & 0 & 0 & 0 & 0 & 0 & 0 & 0 & 0 & 0 & 0 & 29.6 & 59.4 \\
\hline $2^{\text {nd }}$ & 125 & 0 & 4 & 4 & 3.20 & 2 & 100 & 0 & 0 & 0 & 0 & 2 & 30.7 & 64.0 \\
\hline $3^{\text {rd }}$ & 340 & 2 & 5 & 7 & 2.06 & 3 & 60 & 2 & 40 & 0 & 0 & 5 & 30.5 & 64.1 \\
\hline $4^{\text {th }}$ & 548 & 2 & 4 & 6 & 1.09 & 3 & 75 & 1 & 25 & 0 & 0 & 4 & 31.4 & 62.6 \\
\hline $5^{\text {th }}$ & 814 & 5 & 15 & 20 & 2.46 & 8 & 61.54 & 3 & 23.08 & 2 & 15.38 & 13 & 31.3 & 59.7 \\
\hline Aug., $1^{\text {st }}$ & 940 & 14 & 22 & 36 & 3.83 & 14 & 58.33 & 6 & 25 & 4 & 16.67 & 24 & 29.5 & 63.3 \\
\hline $2^{\text {nd }}$ & 1225 & 40 & 65 & 105 & 8.57 & 46 & 62.16 & 18 & 24.33 & 10 & 13.51 & 74 & 27.5 & 63.4 \\
\hline $3^{\text {rd }}$ & 921 & 32 & 22 & 54 & 5.86 & 22 & 53.66 & 7 & 17.07 & 12 & 29.27 & 41 & 29.8 & 68.3 \\
\hline $4^{\text {th }}$ & 514 & 28 & 24 & 52 & 10.12 & 16 & 53.33 & 6 & 20 & 8 & 26.67 & 30 & 28.4 & 61.0 \\
\hline Sep., $1^{\text {st }}$ & 720 & 30 & 25 & 55 & 7.64 & 18 & 51.43 & 8 & 22.86 & 9 & 25.71 & 35 & 28.5 & 61.6 \\
\hline $2^{\text {nd }}$ & 950 & 42 & 66 & 108 & 11.37 & 50 & 68.49 & 13 & 17.81 & 10 & 13.70 & 73 & 28.3 & 61.8 \\
\hline $3^{\text {rd }}$ & 645 & 31 & 22 & 53 & 8.22 & 23 & 67.65 & 7 & 20.59 & 4 & 11.76 & 34 & 28.4 & 62.0 \\
\hline $4^{\text {th }}$ & 580 & 18 & 24 & 42 & 7.24 & 12 & 60 & 5 & 25 & 3 & 15 & 20 & 29.3 & 63.2 \\
\hline Oct., $1^{\text {st }}$ & 840 & 38 & 27 & 65 & 7.74 & 28 & 62.22 & 9 & 20 & 8 & 17.78 & 45 & 27.1 & 65.5 \\
\hline $2^{\text {nd }}$ & 224 & 6 & 5 & 11 & 4.91 & 3 & 60 & 2 & 40 & 0 & 0 & 5 & 29.2 & 66.5 \\
\hline $3^{\text {rd }}$ & 115 & 0 & 0 & 0 & 0 & 0 & 0 & 0 & 0 & 0 & 0 & 0 & 25.6 & 62.8 \\
\hline Total & 9598 & 288 & 330 & 618 & 84.31 & 248 & 61.24 & 87 & 21.48 & 70 & 17.28 & 405 & & \\
\hline Mean \pm SE & $\begin{array}{c}533.22 \pm \\
88.95\end{array}$ & & & & $\begin{array}{c}4.68+ \\
0.89\end{array}$ & $\begin{array}{c}13.78 \pm \\
3.62\end{array}$ & $\begin{array}{c}49.66 \pm \\
6.91^{-}\end{array}$ & $\begin{array}{c}4.83+ \\
1.19\end{array}$ & $\underset{3.05}{17.82 \pm}$ & $\begin{array}{c}3.89+ \\
1.03\end{array}$ & $\begin{array}{c}10.30 \pm \\
2.48\end{array}$ & $\begin{array}{c}22.5+ \\
5.69\end{array}$ & & \\
\hline
\end{tabular}


In the second season, 2011, (Table , 5), Pteromalidae was present from the $5^{\text {th }}$ week of July to the first week of October recording three peaks of population density 12,10 and 8 individuals/ sample on $3^{\text {rd }}$ week of August, $2^{\text {nd }}$ week of September and first week of October at means $29.8,28.3$ and $27.1^{\circ} \mathrm{C}$ and $68.3,61.8$ and $65.5 \%$ R.H., respectively.

\section{Biological studies}

Life cycle of $L$. fabarum on nymphs of $A$. craccivora on faba bean and cowpea at 10,20 and $30^{\circ} \mathrm{C}$.

Table (6) showed that temperature played a important role influencing the period of development of $L$. fabarum on $A$. craccivora. Generally, developmental periods of different stages of the parasitoid were shortened by temperature increased from 10 to $30^{\circ} \mathrm{C}$. At $10^{\circ} \mathrm{C}$, the incubation period of egg averaged 4.98 days, the larval period 8.28 days, while the pupal stage recorded 8.33 days. The total developmental periods was 21.54 days.

At $20^{\circ} \mathrm{C}$, the incubation period of eggs was 2.82 days, larval period was 5.09 days, while pupal stage recorded 5.06 days. The total developmental periods averaged 12.42 days.

Correspondent periods at $30^{\circ} \mathrm{C}$, were $1.97,3.24$ and 3.61 , respectively. The average of total developmental period of the parasitoid L. fabarum lasted 8.81 days. These results agree with those of Tone and Nakashima (2008).

Table (5) showed that the adult longevity was longest at $10^{\circ} \mathrm{C}$, and also that of females was longer than males at the three temperatures $\left(10,20\right.$ and $\left.30^{\circ} \mathrm{C}\right)$. Recorded longevities of males and females were $6.30 \& 8.73,4.18 \& 6.17$ and $3.15 \& 4.20$ days at the three temperatures, respectively.

\section{Sex ratio}

A total number of 340 L. fabarum adults emerged from $490 \mathrm{~A}$. craccivora mummies showing $69.39 \%$ emergence. The number of females was 176 and 164 for males. The sex ratio was 1.073 females: 1 male.

Table 6. Developmental periods of immature stages and longevity of adults in days of the parasitoid Lysiphlebus fabarum on $A$. craccivora infesting faba bean plants at 10,20 and $30^{\circ} \mathrm{C}$ and $60 \pm 5 \%$ R.H.

\begin{tabular}{|c|c|c|c|}
\hline Temp. ${ }^{\circ} \mathrm{C}$ & 10 & 20 & 30 \\
\hline Stage Duration & mean $\pm S . E$ & mean $\pm S . E$ & mean \pm S.E \\
\hline Egg & $4.98 \pm 0.15$ & $2.82 \pm 0.09$ & $1.97 \pm 0.02$ \\
\hline Larva & $8.28+0.27$ & $5.09+0.06$ & $3.24 \pm 0.07$ \\
\hline Pupa & $8.33+0.15$ & $5.06 \pm 0.11$ & $3.61 \pm 0.07$ \\
\hline Immature stages & $21.54+0.26$ & $12.42 \pm 0.10$ & $8.81 \pm 0.13$ \\
\hline \multicolumn{4}{|l|}{ Longevity: } \\
\hline$\hat{0}$ & $6.3 \pm 0.37$ & $4.18 \pm 0.05$ & $3.15 \pm 0.06$ \\
\hline q & $8.73+0.22$ & $6.17 \pm 0.16$ & $4.20 \pm 0.04$ \\
\hline
\end{tabular}




\section{ACKNOWLEDGEMENTS}

Deep thanks to Prof. Dr. A. El-Heneidy, Biological Control Department, Plant Protection Research Institute, Agricultural Research Center, Giza, Egypt. For identification of the parasitoid species.

\section{REFERENCES}

1. Abdel-Samad, S.S.M., 1996. Studies on natural enemies on certain insects attacking leguminous crop. M.Sc. Thesis, Fac. of Agric. Ain Shams Univ., 94 pp.

2. Abdel-Samad, S.S.M. and M.A. Ahmed. 2006. Population fluctuations of Aphis craccivora and Liriomyza trifolii and their endoparasitoids on certain faba bean varieties . Ann. Agric. Sci. Cairo 51 (2): 531-540.

3. Abou-Attia, F.A., H.A. Boraei, F.M. El-Agamy and Fathia, A.K. Salem. 2001. Survey and population fluctuation of primary and secondary parasitoids of some aphid species on four crops and parasitoid of Coccinella undecimpunctata at Kafr ElSheikh, Proceedings, the $1^{\text {st }}$ Int. Egyptian Romanian Conf., Zagazig, Egypt., Dec., 6-8 ${ }^{\text {th }}, 2003$ 61-76.

4. Costat Software 1990. Micro Computer Programe Analysis Version 4.20, Co Hort Software, Berkeley, C.A.

5. Cruz, Boelpaepe, M.O., M.R. Forra, J.E., Fernandes and C.M O. Boelpaepe 1992. Aphid pests of vegetable crops and aphidophagous insects caught in a suction trap in the Algarve, Portugal. Bull. OILB. SROP 15 (4): 16-27.

6. El-Defrawi G.M., Azza Emam, I.A. Marzouk and L. Rizkalla. 2000. Population dynamics and seasonal distribution of Aphis craccivora Koch. and associated natural enemies in relation to virus disease incidence in faba bean fields. Egypt. J. Agric. Res., 78 (2): 627-641.

7. Farrell, J.A. and M.W. Stufkens. 1990. The impact of Aphidius rhopalosiphi (Hymenoptera: Aphidiidae) on population of the rose grain aphid (Metopolophium dirhodum) (Homoptera: Aphididae) on cereals in Cankrbury, New Zeland. Bull. Ent. Res., 80 : 377-383.

8. Maghraby, H.M.M. 2012. Studies on the parasitoid Diaeretiella rapae on some aphid species in sharkia Governorate, M.Sc. Thesis, Fac. of Agric., Moshtohor, Benha Univ., pp.222.

9. Ragab, M.E. 1996. Biology and efficiency of Trioxys angelicae Hal. (Hymenoptera: Aphidiidae), a newly recorded parasitoid of Aphis craccivora Koch. (Homoptera: Aphidiidae). Egypt, J. Biol. control, 6 (1): 7-11. 
10. Ragab, M.E., A.A. Abou El-Naga, A.A. Ghanim and A.A. Saleh. 2002a. Effect of host aphid species, temperature and food supple on some biological, characteristics of the two aphid parasitoids, Diaeretialla rapae (M'Intosh) and Aphidius sp. (Hymenoptera: Aphidiidae). J. Agric. Sci. Mansoura Univ., 27 (7): 4997-5002.

11. Ragab, M.E., A.A. Abou El-Naga, A.A. Ghanim and A.A. Saleh. 2002b. Ecological studies on certain aphid parasitoids, especially those of Aphis craccivora Koch. J. Agric. Sci. Mansoura Univ., 27 (4): 2611-2620.

12. Saleh, A.A.A. 2004. Mass production and field application of some aphid natural enemies . Ph. D. Thesis, Fac. of Agric., Mansoura Univ., pp. 161.

13. Saleh, A.A.A., W.M.H. Desuky and Nadia E. Mohamed 2009. Studies on some parasitoids of the cowpea aphid Aphis craccivora Koch. (Homoptera: Aphididae) in Egypt. Egypt, J. Biol. Pest. Cont. 19 (1): 11-16.

14. Sarhan, A.A. 1976. Studies on the biological control of cotton white fly Bemisia tabaci (Genn.) in Egypt. M. Sc. Thesis Fac. of Agric. Cairo Univ., 164. pp.

15. Selim, A.A., S.A. El-Refai and A.El-Gantiry. 1987. Seasonal fluctuations in the population of Aphis craccivora Koch., Myzus persicae (Sulz.) Aphis gossypi (Glov.) and their parasites. Ann. Agric. Sci. Ain Shams Univ., 32 (3): 1837-1848.

16. Stary, P. 1976. Aphid Parasites (Hymenoptera: Aphidiidae) of the Mediterranean area. Transactions of the Czechoslovak Academy of Sciences, Series of Mathematical and Natural Sciences, 86:1-95.

17. Stary, P. and C. Erdelen. 1987. Aphid parasitoids (Hym., Aphidiidae, Aphelinidae) from the Yemen Arab Republic. Entomol., 27 (1): 105-108.

18. Stary, P. and D. H Stechmann. 1990. Ephedrus cerasicola stary (Hym., Aphidiidae), a new biocontrol agent of the banana aphid Pentalonia nigronervosa Coq. (Hom., Aphididae). J. Appl., Ent., 109: 457-462.

19. Tone, J. and Y. Nakashima. 2008. Development and reproductive characteristics of Lysiphlebus fabarum (Marshall) (Hymenoptera: Aphidiidae), a parasitoid of cowpea aphid, Aphis craccivora Koch. (HomopteraL Aphididae). Japanese J. Appl. Ent. Zool., 52 (3): 149-151.

20. Youssif, M.A. I. 2009. Studies on aphidophagous insects of Aphis craccivora Koch. M.Sc. Thesis, Fac. of Agric., Zagazig Univ., 134pp. 


\section{نسب التطفل على من اللوبيا Aphis craccivora Koch. و اللوبيا فى الاراضى المستصلحة حليثا فى مصر لمر

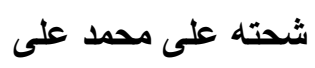

معهُ بحوث وقاية النباتات ، مركز البحوث الزرراعية ، الدقى ، الجيزة ، مصر

تم در اسة الكثافة العددية لمن اللوبيا Aphis craccivora Koch الذي يصيب نباتات الفول البلدى

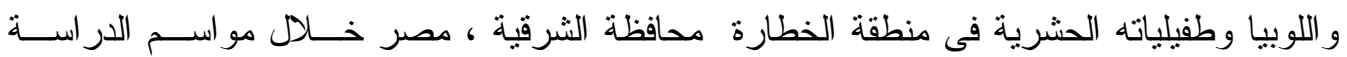

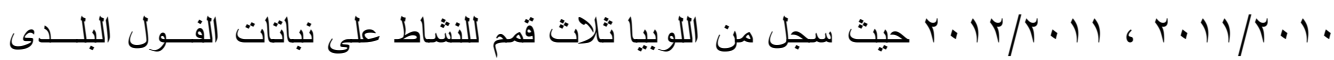

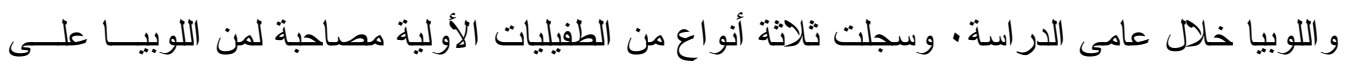
الفول البلاى هى :

Diaerettiella rapae (M'Intosh), Aphidius colemani Viereck and Ephedrus sp.

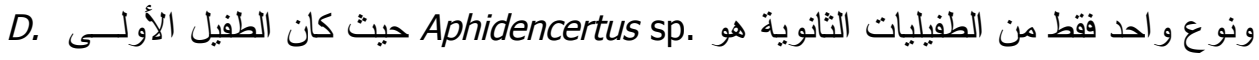

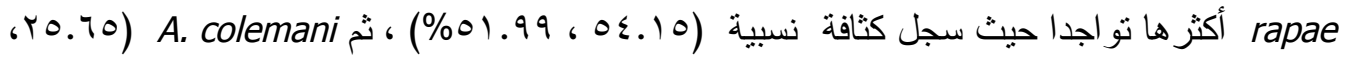

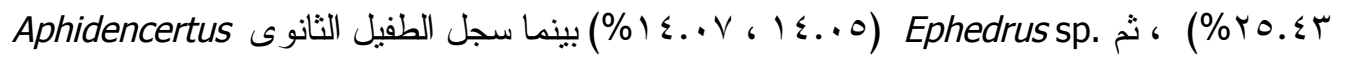

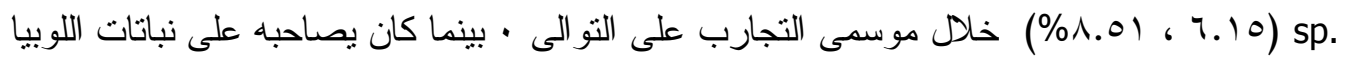

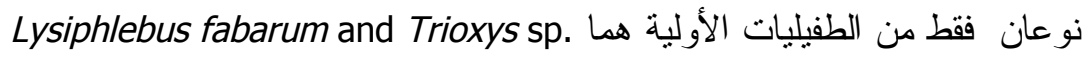

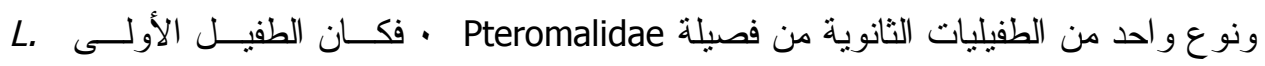
أكثر ها تو اجداً بكثافة نسـبية (r. Thabarum

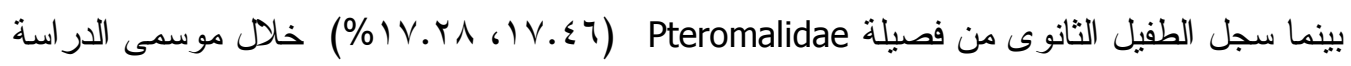

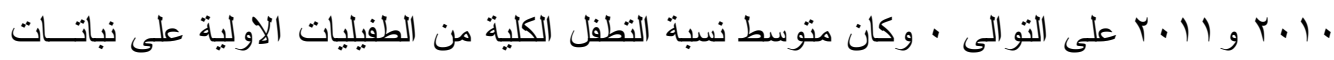

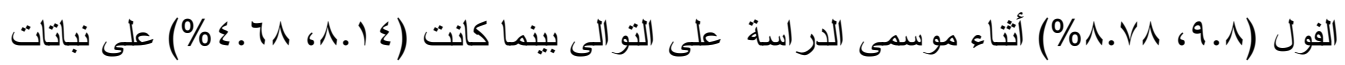

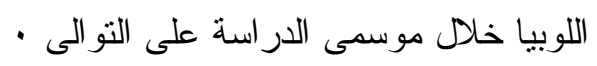

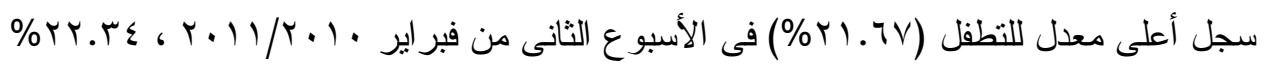

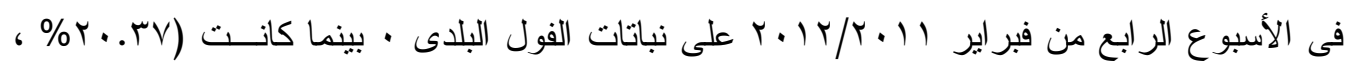

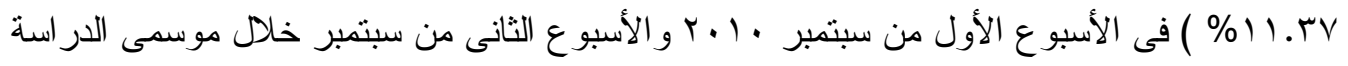

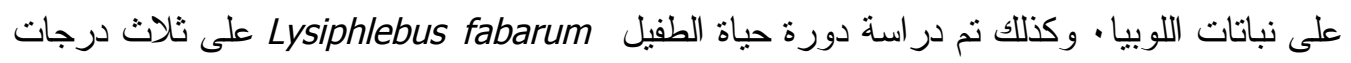

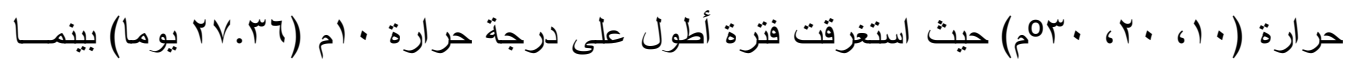

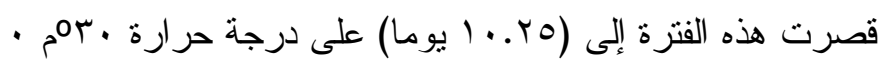

